\title{
Characterization of the double-double bend achromat lattice modification to the Diamond Light Source storage ring
}

\author{
I. P. S. Martin, ${ }^{*}$ M. Apollonio, R. T. Fielder, E. Koukovini-Platia, and B. Singh \\ Diamond Light Source, Oxfordshire, OX11 ODE, United Kingdom \\ R. Bartolini \\ Diamond Light Source, Oxfordshire, OX11 ODE, United Kingdom and John Adams Institute, \\ University of Oxford, Oxford OX1 3RH, United Kingdom
}

(Received 8 December 2017; published 25 June 2018)

\begin{abstract}
The Diamond storage ring has recently been modified by replacing one of the original double bend achromat (DBA) cells with a double-DBA (DDBA) cell. The new cell marks a fundamental break to the machine symmetry, and the final engineered solution shares many design features in common with modern multi-bend achromat cells. In this paper we review the impact that the modification has had on the linear and nonlinear optics, collective effects, and overall performance. Where appropriate, comparison to model predictions are also made.
\end{abstract}

DOI: 10.1103/PhysRevAccelBeams.21.060701

\section{INTRODUCTION}

The need to improve the provision of tailored, highbrightness photon beams to an ever-increasing number of experimental users has driven many of the recent developments at synchrotron radiation facilities worldwide. The majority of these beamlines use magnetic insertion devices (IDs) to deflect the electron beams and thus provide a tuneable source of synchrotron radiation. Third generation light sources are typically designed with a regular, periodic structure, in which cells of magnets are alternated with the straight sections that house the IDs. This high degree of periodicity helps to maintain a long lifetime and good injection efficiency, as many of the main structural resonances of the lattice become suppressed. This cancellation can be compromised by the presence of field and alignment errors, however, there are clear operational benefits in keeping the machine periodicity high [1].

In recent years, several light source facilities have made modifications to the lattice which break this periodicity. These range from being of relatively minor impact (such as the installation of high-field IDs), to more significant modifications such as the replacement of individual dipoles with super-bends to improve the radiation spectrum [2-4] or the installation of double mini-beta cells to allow two small-gap insertion devices to be located in a single straight

\footnotetext{
*ian.martin@diamond.ac.uk

Published by the American Physical Society under the terms of the Creative Commons Attribution 4.0 International license. Further distribution of this work must maintain attribution to the author(s) and the published article's title, journal citation, and DOI.
}

section [5]. In all these examples, steps have been taken either to compensate for the optics distortions locally or to introduce the changes such that the periodicity between cells is maintained.

Since user operations began in 2007, Diamond Light Source has followed a similar pattern of development. The original design of the storage ring consisted of twentyfour double-bend achromat (DBA) cells with a six-fold symmetry and an initial complement of seven IDs [6,7]. Since then, an additional twenty-two IDs have been installed [8], and two long straight sections have been modified to provide double minibeta with horizontal focusing optics [9]. The most significant change however occurred in October 2016 with the replacement of one of the DBA cells with a double-DBA (DDBA) cell $[10,11]$. The initial concept for the DDBA cell was developed as a potential lattice upgrade for the entire Diamond storage ring [12], however, the primary purpose of the single-cell replacement was to create space in the centre of the arc for an additional in-vacuum ID. This modification was anticipated to have a significant impact on the machine performance, constituting a fundamental break to the machine symmetry.

The integration of the DDBA cell into the existing storage ring has presented a number of challenges. Due to the need for high-gradient quadrupole and sextupole magnets (up to $70 \mathrm{~T} / \mathrm{m}$ and $1600 \mathrm{~T} / \mathrm{m}^{2}$ respectively), the new cell is much closer to modern multi-bend achromat (MBA) cells in design than the original DBA cells [13-15]. This in turn has caused a number of engineering issues that needed to be addressed, such as the first use of gradient sector bends in the Diamond storage ring, the reduced physical apertures needed for the high-gradient magnets, 
and the mix of stainless steel and copper vacuum chambers needed to cope with the synchrotron radiation heat load. From an optics point of view, the need to match the DDBA cell to the adjacent DBA cells means the optics are not ideally suited either to emittance reduction or for establishing the correct phase advance for optimizing the nonlinear beam dynamics. The fundamental break in the machine symmetry also makes the new machine significantly more sensitive to field and alignment errors. Taking all of these factors into account, the DDBA lattice modification presents a unique opportunity to study the performance of this novel cell design, to understand how well standard modeling codes can predict the final performance of complex, nonsymmetric rings, as well as to quantify the relative impact a modern MBA cell design can have on machine impedance.

\section{THE DDBA CELL}

The primary constraint when designing the DDBA cell was that it should fit within the existing storage ring and beam-line footprints, whilst providing an additional $3.4 \mathrm{~m}$ long, low dispersion straight section in the centre of the arc suitable for an in-vacuum ID. The new cell is described in detail in [11], where a discussion on the significant design choices can also be found. For convenience, the storage ring parameters before and after installation are provided in Table I, and a plot of the Twiss and dispersion functions are shown in Fig. 1.

Examination of the optical functions for the new cell reveals a small residual asymmetry remaining across the new straight section. In addition, the location of the horizontal waists in the adjacent straights have shifted towards the new DDBA cell. This was found to be necessary in order to keep the beta functions low within the DDBA cell and minimize particle loss on the new, narrower apertures. Note also that the DDBA cell does not
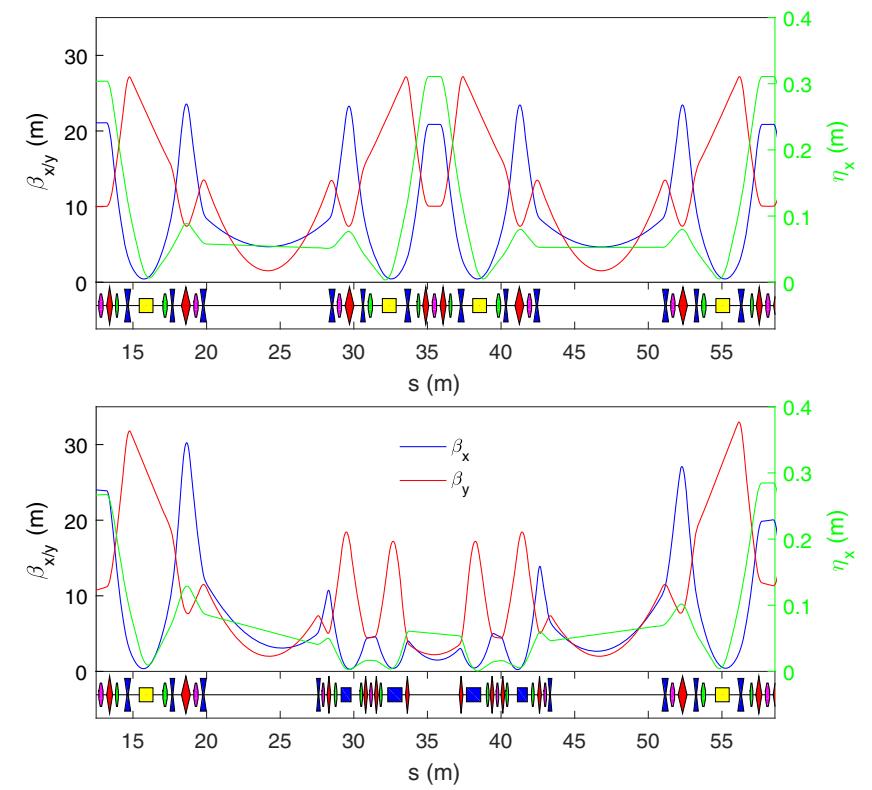

FIG. 1. Plot of optics functions and layout before (top) and after (bottom) installation.

sit in the center of the original four-cell super-period, contributing toward the asymmetry.

\section{MACHINE CHARACTERIZATION}

\section{A. Linear optics}

Control of the linear optics is fundamental to the successful operation of any electron storage ring. Many techniques exist to do this, the majority of which use either the turn-by-turn beam position monitor (BPM) or closedorbit response matrix data to extract information on the underlying machine optics [16]. At Diamond, the standard technique is to use a modified version of the Linear Optics from Closed Orbit (LOCO) algorithm which has been optimized for speed of execution $[17,18]$.

TABLE I. Comparison of main lattice parameters before and after the installation of the DDBA cell.

\begin{tabular}{lcc}
\hline \hline Parameter & Pre-DDBA & Post-DDBA \\
\hline Lattice & $24 \times \mathrm{DBA}$ & $1 \times$ DDBA $+23 \times \mathrm{DBA}$ \\
Circumference & $561.600 \mathrm{~m}$ & $561.571 \mathrm{~m}$ \\
Periodicity & 6 & 1 \\
Harmonic number & 936 & 936 \\
Energy & $3 \mathrm{GeV}$ & $3 \mathrm{GeV}$ \\
Horizontal emittance & $2.52 \mathrm{~nm} \mathrm{rad}$ & $2.70 \mathrm{~nm} \mathrm{rad}$ \\
Vertical emittance & $8.0 \mathrm{pm} \mathrm{rad}$ & $8.0 \mathrm{pm} \mathrm{rad}$ \\
Energy spread & $9.63 \times 10^{-4}$ & $9.60 \times 10^{-4}$ \\
Tunes $\left(Q_{x} / Q_{y}\right)$ & $27.210 / 13.364$ & $28.172 / 13.273$ \\
Natural chromaticity $\left(\xi_{x} / \xi_{y}\right)$ & $-79.4 /-38.1$ & $-78.8 /-41.2$ \\
Operating chromaticity $\left(\xi_{x} / \xi_{y}\right)$ & $2.0 / 2.0$ & $1.5 / 2.0$ \\
Energy loss per turn $($ without IDs $)$ & $1.005 \mathrm{MeV}$ & $0.988 \mathrm{MeV}$ \\
Momentum compaction factor $\left(\alpha_{1} / \alpha_{2}\right)$ & $1.60 \times 10^{-4} / 1.78 \times 10^{-3}$ & $1.57 \times 10^{-4} / 1.70 \times 10^{-3}$ \\
Maximum $\beta_{x} / \beta_{y}$ & $31.52 \mathrm{~m} / 29.66 \mathrm{~m}$ & $31.57 \mathrm{~m} / 33.74 \mathrm{~m}$ \\
\hline \hline
\end{tabular}




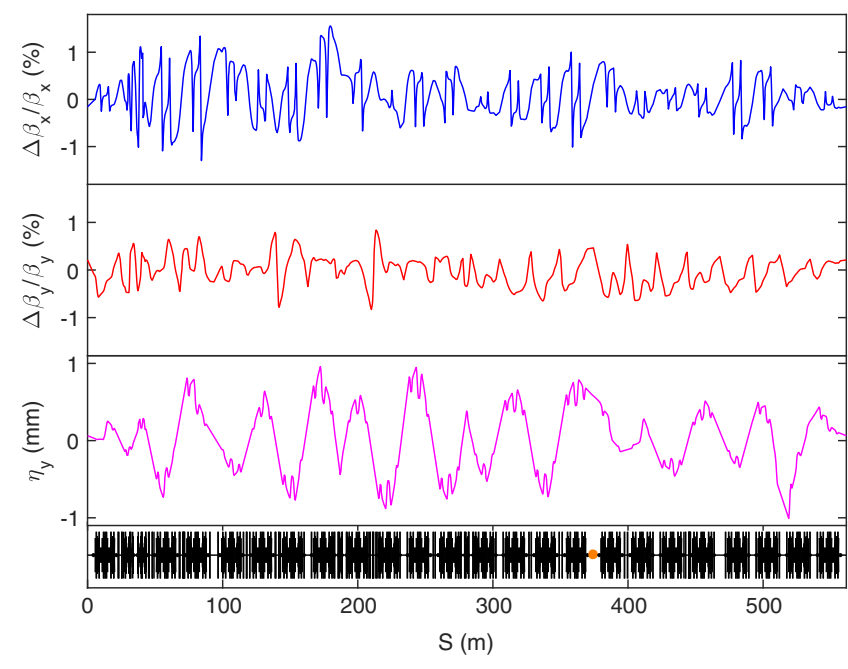

FIG. 2. Measured optics distortions after correction.

In order to use LOCO after the installation of the DDBA cell, several changes had to be made to the default set-up. The first of these was to enable the new gradient sector bending magnets to be included in the fit. Within the DDBA cell there are two long and two short dipoles, each of which contain a defocusing quadrupole component of $k=-1.43 \mathrm{~m}^{2}$. This can be altered by changing the magnet excitation, however, doing so will also change the integrated bending angle, and so can only be carried out in combination with a physical shift of the magnet to compensate [11].

Studies using the machine model before the installation also highlighted a possible problem with fitting the pairs of focusing quadrupoles in the dispersion bumps between the dipoles. The phase advance between these magnets is small with only a limited number of BPMs and correctors in the vicinity, making the individual gradients difficult to determine. As such, the fitted gradients were found to diverge under certain conditions. Tests were carried out to see if pairing the magnets would help, but adding penalty weights that restrict the variation between these families was found to be more effective.

Having established a suitable configuration for the LOCO fit, further studies were carried out to determine an optimal value for the singular value decomposition (SVD) threshold. This value was set to provide the best compromise between minimizing the $\chi^{2}$ of the fit between measured and model response matrix, whilst also reducing the sensitivity to measurement noise. Since the installation, measurements and correction of the linear optics have been carried out routinely, an example of which is shown in Fig. 2. This data was measured with IDs fully open and confirms that the linear optics are well controlled, with peak beta-beat around $1 \%$ in both planes and vertical dispersion below $1 \mathrm{~mm}$.

During commissioning, a problem arose when setting the skew quadrupole gradients in the DDBA cell. As with the

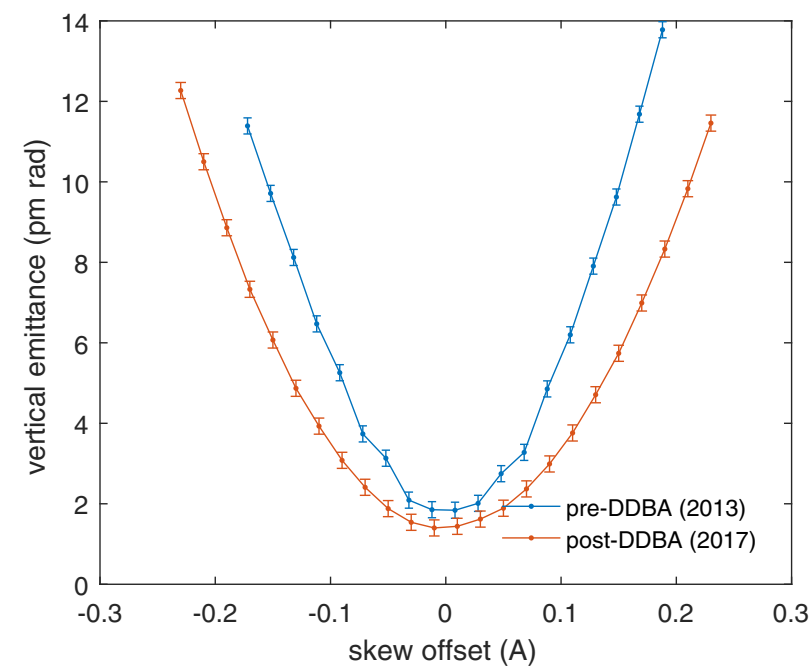

FIG. 3. Vertical emittance as a function of skew quadrupole offset before and after the installation of the DDBA cell. The error bars for the measured data are estimates based on imprecise knowledge of the pinhole camera point-source functions and Twiss parameters in the dipoles.

focussing quadrupole pairs between the dipoles, the adjacent pairs of skew quadrupoles were found to diverge, particularly when the local ID was closed. To avoid this problem and to prevent the magnets saturating, the LOCO configuration was altered to treat the skew-quadrupole pairs as single magnets. This solved the problem in the short-term, bringing down the strengths of the magnets substantially. Scans of vertical emittance [19] as a function of skew quadrupole strength confirms excellent coupling control has been maintained, as shown in Fig. 3. Since all ten sextupoles in the DDBA cell are equipped with skewquadrupole coils but only six are powered, the option of reconfiguring the choice of magnets remains as a possible long-term solution.

\section{B. Nonlinear optics}

The first measurements of the nonlinear beam dynamics concentrated on measuring the dynamic aperture and frequency map for the bare lattice (i.e., without IDs). This was done using two single-turn pinger magnets (one horizontal and one vertical) located at the end of an ID straight. The filling pattern was kept short (100 bunches out of a possible 936) in order to produce a uniform kick to all bunches, and the total beam current was set to $10 \mathrm{~mA}$. This current gave a good compromise between good signal strength for the BPMs whilst minimizing the influence of collective effects. The amplitude of kick in each plane was increased in steps until the whole beam was lost, and for each kick the turn-by-turn data was recorded from all BPMs for post-processing. The method of converting the raw BPM button data into actual beam position on each turn is described in [20], along with the method for 


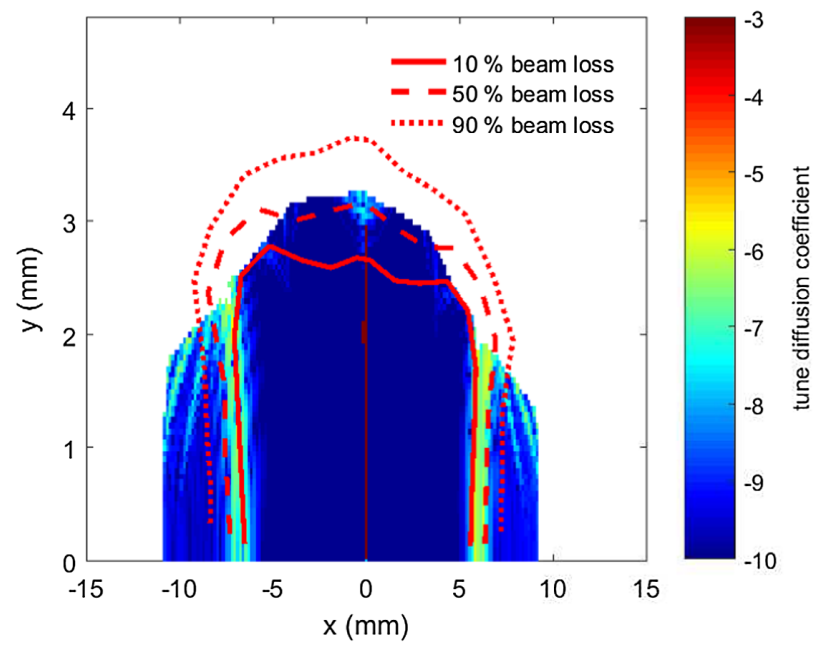

FIG. 4. Measured dynamic aperture following the installation of the DDBA cell (red lines). For comparison, the dynamic aperture computed using the machine model is also shown.

projecting these amplitudes back to the injection point using the model.

The dynamic aperture for one such measurement is shown in Fig. 4. In this plot, three boundaries indicating different levels of beam loss are shown in red, highlighting the fact that the edge of the dynamic aperture is not particularly well defined. These lines have been superimposed over the dynamic aperture computed from the machine model using the ELEGANT tracking code [21]. This model uses first-order edge focussing and measured systematic multipole errors in the dipoles, fringe fields in the quadrupoles and has the sextupole strengths scaled to $3.015 \mathrm{GeV}$ [22]. The linear chromaticity in the model has also been adjusted to $1.5 / 2.0$ to be consistent with the measurements. Comparing the measured dynamic aperture to the model prediction, it is clear that the model overestimates the final aperture by several $\mathrm{mm}$ in the horizontal plane. The measurements show strong beam loss at $\pm 6 \mathrm{~mm}$, which coincides with the beam crossing the $6 Q_{x}=169$ resonance. The agreement in the vertical plane is much closer, as this is dominated by the physical apertures.

The measured and model frequency maps corresponding to the dynamic apertures shown in Fig. 4 are shown in Fig. 5. Here there is reasonable qualitative agreement, although the vertical detuning with horizontal amplitude is slightly negative rather than slightly positive (corresponding to a difference of $\Delta Q_{y} \approx 3 \times 10^{-3}$ at $6 \mathrm{~mm}$ horizontal amplitude).

The dependence of the nonlinear beam dynamics on momentum deviation has been assessed by recording the betatron tunes as a function of rf frequency (see Fig. 6). This was again carried out with a total of $10 \mathrm{~mA}$ in 100 bunches stored in the ring, with the maximum deviation taken to be the first point of beam loss. Conversion of the rf frequency shift to momentum deviation has been done using the equation

$$
\frac{\Delta p}{p}=\frac{1}{2 \alpha_{2}}\left(-\alpha_{1}+\sqrt{\alpha_{1}^{2}-4 \alpha_{2} \frac{\Delta f_{r f}}{f_{r f}}}\right)
$$

with the values for $\alpha_{1}$ and $\alpha_{2}$ taken from Table I. There is excellent agreement between the measured horizontal detuning with momentum and the model prediction out to $\pm 2 \%$. The vertical tune shift again shows a good qualitative agreement, although there appears to be a stronger second-order chromaticity component in the model than appears in the machine. The origin of this
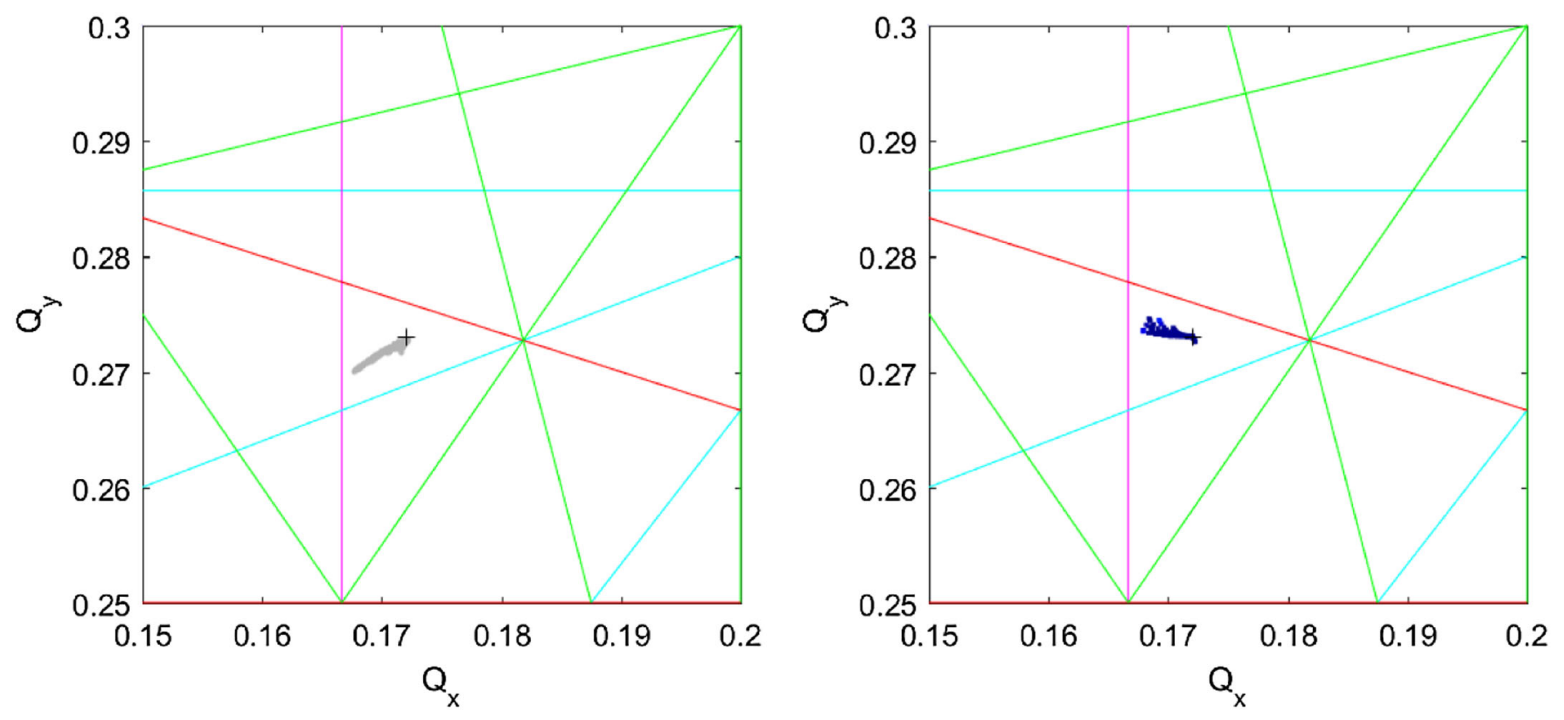

FIG. 5. Comparison between frequency maps measured on the machine (left) and computed from the model (right) over the measured dynamic aperture. The nominal tune-point is marked as ' + '. 

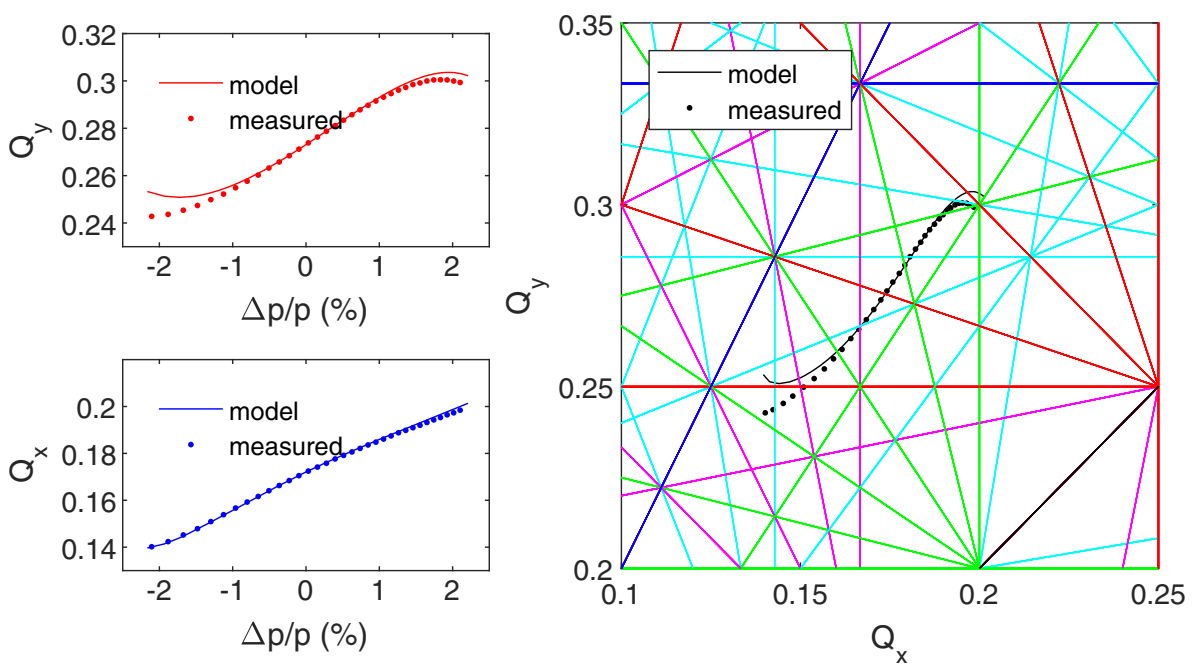

FIG. 6. Comparison of tune-shift with momentum between model and measurement.

discrepancy (and that of the vertical detuning with horizontal amplitude) is believed to originate in the way the dipole fringe fields are described and is the subject of ongoing investigations. Using Eq. (1), the momentum aperture is measured to be $\pm 2.1 \%$ to the first point of beam loss, compared to $-1.4 \% /+2.8 \%$ for the original lattice [23].

\section{Tune scans}

The relative strength of the resonances appearing in the lattice can be conveniently probed by recording injection efficiency as a function of tune point. This is shown in Fig. 7 for both the machine and the ELEGANT model. In both cases, studies were performed for the bare lattice (no IDs), with the tune point first set for the stored beam before determining the injection efficiency. For the model, tracking was carried out over 6000 turns (one transverse damping time) using the same model as described in Sec. III B. The injected beam was offset from the stored beam by $-7.5 \mathrm{~mm}$, based on the most recent data available.

The two injection efficiency maps show many features in common. In both cases, the $2 Q_{x}+2 Q_{y}=83$ octupole resonance has the strongest impact on injection efficiency (diagonal red line in upper half of plots). This type of error is known to be driven by the quadrupole fringe fields, and also exists as a multipole error in the original DBA quadrupole magnets. The next most prominent line is the leading order skew sextupole resonance $Q_{y}-2 Q_{x}=$ -43 (diagonal blue line in top left of plots). This is mainly driven by tilt errors in the normal sextupoles. The dominant decapolar resonances appearing are the $5 Q_{x}=$ 141 and $2 Q_{y}-3 Q_{x}=-58$ lines (vertical green line and diagonal green line running from bottom left to top right respectively). Normal decapole errors were included in the ELEGANT model as systematic errors in the dipoles, but the resonances are also found to be driven in the ideal, errorfree case. Since these lines appear much weaker in the measured data, this suggests the applied systematic errors in the model are overly pessimistic. Finally, the $6 Q_{x}=$ 169 duodecapole resonance (vertical magenta line) is weakly excited in both measured and model data. As with the $5 Q_{x}=141$ resonance, the drop in injection efficiency appears displaced with respect to the resonance itself. This is due to the tune-shift with amplitude of the injected beam.

The operational tune point of $Q_{x}=28.172, Q_{y}=$ 13.273 is marked as a black dot in Fig. 7. Despite the fact that the injection efficiency for the bare lattice is higher elsewhere, this is not the case for the lifetime. In addition, the performance at any given tune point can vary considerably depending upon the specific combination of ID gaps and phases. Overall, the lifetime and injection efficiency are found to be largely constant at the selected tune point under most conditions, making it suitable for operations.

\section{Impact on Touschek lifetime}

The Touschek lifetime is commonly computed using the Piwinski formula [24],

$$
\frac{1}{\tau}=\left\langle\frac{r_{e}^{2} c N_{p}}{8 \pi \gamma^{2} \sigma_{s} \sqrt{\sigma_{x}^{2} \sigma_{y}^{2}-\sigma_{E}^{4} \eta_{x}^{2} \eta_{y}^{2}} \tau_{m}} F\left(\tau_{m}, B_{1}, B_{2}\right)\right\rangle
$$

where $\tau_{m}$ is related to the local momentum acceptance $\epsilon_{a}(s), N_{p}$ is the number of particles per bunch, $r_{e}$ is the classical electron radius, $\gamma$ is the Lorentz factor, $\sigma_{s}, \sigma_{x}$ and $\sigma_{y}$ are the bunch dimensions, $\sigma_{E}$ is the relative energy 

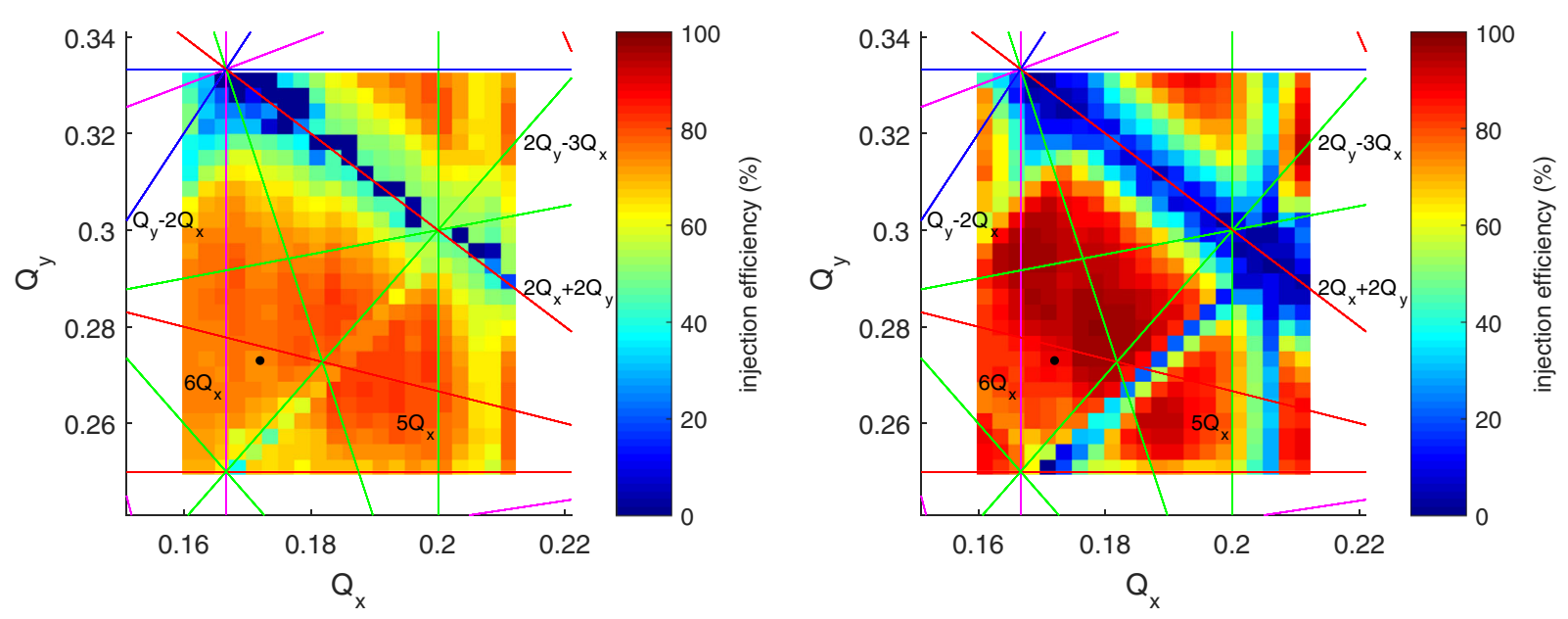

FIG. 7. Injection efficiency vs tune point measured after the installation of the DDBA cell (left) and a comparison to the predicted map found using the ELEGANT model (right).

spread and $\eta_{x}$ and $\eta_{y}$ are the horizontal and vertical dispersion functions. $F\left(\tau_{m}, B_{1}, B_{2}\right)$ is a form factor encapsulating how the scattering rate varies around the ring, with $B_{1}$ and $B_{2}$ parameters of the local beam sizes and optical functions. The momentum acceptance $\epsilon_{a}(s)$ is typically calculated from the machine model via particle tracking, and varies in both the positive and negative directions due to non-linearities in the momentum compaction factor. $\epsilon_{a}(s)$ also exhibits a transition between being dominated by the rf acceptance at low voltages and by the lattice acceptance at high rf voltage $[25,26]$.

The Touschek lifetime for the new ring has been measured by recording lifetime as a function of rf voltage for the bare lattice. Before recording the data, the storage ring optics were corrected using LOCO (thereby establishing agreement between machine and model to the percentagelevel), and the vertical emittance was determined using the combined X-ray pinhole camera images as described in [19]. The estimated uncertainty in the vertical emittance was $\pm 0.2 \mathrm{pm} \mathrm{rad}$. The contribution from the gas lifetime was compensated for by recording lifetime as a function of vertical collimator aperture [27], then subtracted from the overall measured lifetime.

The final Touschek lifetime for the DDBA lattice is shown in Fig. 8, along with a measurement taken before the DDBA cell was installed. The two curves are found to be offset from each other consistent with the lower energy loss per turn for the new ring. The peak of the curve has also shifted from 2.3 MV down to $2.2 \mathrm{MV}$, and the peak lifetime has reduced by $10 \%$ from $15.4 \mathrm{~h}$ to $14.0 \mathrm{~h}$. Assuming the values for $\alpha_{1}$ and $\alpha_{2}$ given in Table I are correct, the peak at $2.2 \mathrm{MV}$ corresponds to a momentum acceptance of $+3.2 \% /-4.3 \%$.

Also shown in Fig. 8 is a calculation of the lifetime using Eq. (2) (assuming the RF acceptance is dominant), after substituting for the natural bunch length with the measured one (dashed black line). This curve starts to diverge from the measured curve at $1.6 \mathrm{MV}$, corresponding to an $\mathrm{RF}$ momentum acceptance of $+2.1 \% /-2.6 \%$. This value compares well to the momentum acceptance found during the nonlinear chromaticity measurements described in Sec. III B, where the limits were set at the first point of beam loss (rather than the maximum possible energy deviation).

Finally, the measured lifetime curves before and after the DDBA cell installation have been compared to the results of tracking calculations made using the ELEGANT model. Similar to the analytic calculation, the measured bunch lengths have been combined with the tracking data to produce the final curves. In each case, the qualitative agreement between the measured and predicted lifetimes is remarkable.

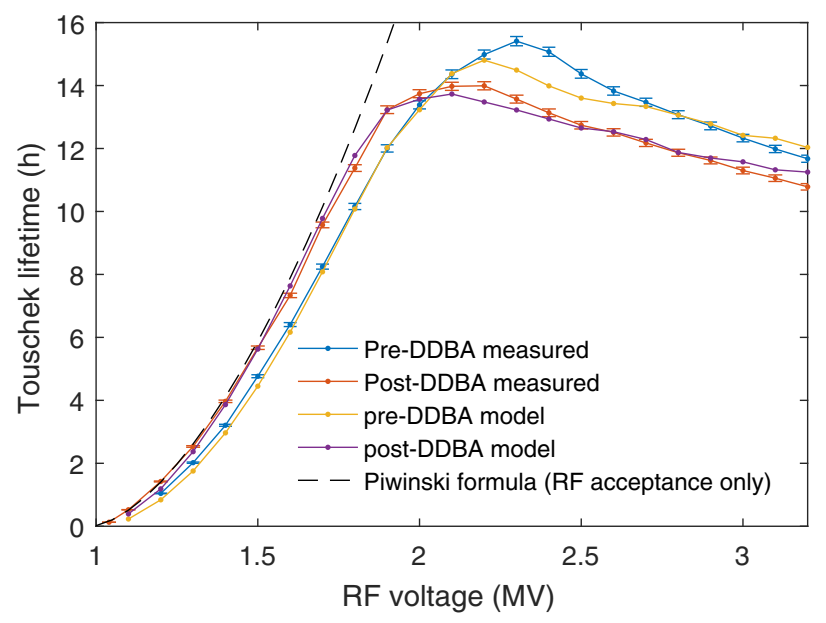

FIG. 8. Comparison of Touschek lifetime vs rf voltage for the bare lattice. Measured data is compared to the results from particle tracking and analytic calculations, both before and after installation. The error bars for the measured data are estimates based on fluctuations observed during data acquisition. 


\section{E. Collective effects}

The impact of the vacuum chamber on beam dynamics has been under study at Diamond for a number of years (see for example [28-30]). These studies have focussed on measuring the current-dependent behaviour of the beam under a variety of conditions, then reproducing this behaviour in simulation by adjusting the parameters of a simplified impedance model. In each case, the overall coupling impedance of the vacuum chamber has been approximated as the sum of geometric and resistive wall (RW) contributions, with the geometric components being modelled as broadband or high-Q resonators. For single bunch simulations, a purely inductive component has also been included in the longitudinal plane to improve the match with the measured data.

In the transverse and longitudinal planes, the equations used to describe the resonator impedances are

$$
Z_{x, y}^{B B R}(\omega)=\frac{\omega_{x, y}}{\omega} \frac{R_{x, y}}{1+i Q\left(\frac{\omega_{x, y}}{\omega}-\frac{\omega}{\omega_{x, y}}\right)}
$$

and

$$
Z_{\|}^{B B R}(\omega)=\frac{R_{\|}}{1+i Q\left(\frac{\omega_{\|}}{\omega}-\frac{\omega}{\omega_{\|}}\right)}
$$

respectively, where $Q$ is the resonator quality factor, $\omega_{x}, \omega_{y}$ and $\omega_{\|}$are the resonator frequencies and $R_{x}, R_{y}$ and $R_{\|}$are the shunt impedances. For short-range wakefields (relevant for single-bunch studies), a single $Q=1$ broadband resonator (BBR) close to the vacuum chamber cut-off frequency $(\omega=2 \pi \times 8.3 \mathrm{GHz})$ has been used to describe the interaction in all three planes.

The contribution from the RW impedance also has transverse and longitudinal components per unit length, given by

$$
\frac{Z_{x, y}^{R W}(\omega)}{L}=(\operatorname{sgn} \omega+i) \frac{1}{\pi b_{x, y}^{3}} \sqrt{\frac{Z_{0} c \rho}{2|\omega|}} G_{1 x, y}
$$

and

$$
\frac{Z_{\|}^{R W}(\omega)}{L}=(1+i \operatorname{sgn} \omega) \frac{1}{2 \pi b_{x, y}} \sqrt{\frac{Z_{0} \rho|\omega|}{2 c}} G_{0}
$$

respectively, where $b_{x, y}$ is the average vacuum chamber half aperture, $Z_{0}$ is the impedance of free space, $c$ is the speed of light and $\rho$ is resistivity of the material. In these studies, the vacuum chamber has been modeled as a single layer of stainless steel $\left(\rho=7.3 \times 10^{-7} \Omega \mathrm{m}\right) . G_{1 x, y}$ and $G_{0}$ are form factors for the vacuum chamber [31].

Finally, the purely inductive impedance used in the longitudinal plane has been calculated using

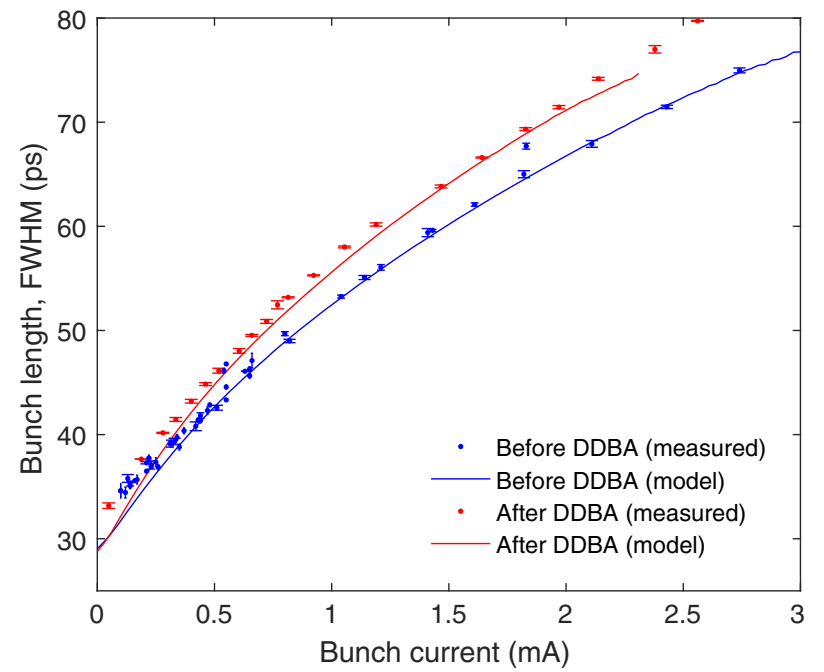

FIG. 9. Full-width at half-maximum (FWHM) bunch length as a function of current. The error bars on the measured data show the standard error over 801 samples.

$$
Z_{\|}^{\text {ind }}(\omega)=i L \omega
$$

where $L$ is the inductance.

Shown in Fig. 9 is the full-width at half-maximum (FWHM) bunch length vs current before and after the installation of the DDBA cell, measured using an Optronis dual-sweep streak camera [32]. All measurements were taken at a fixed rf voltage of $2.5 \mathrm{MV}$, insertion devices closed and with the transverse multi-bunch feedback (TMBF) switched off. Examination of the two data sets shows a clear increase in the bunch lengthening caused by the new cell. The simulated bunch lengths produced by SBTRACK [29] using the simplified impedance model are also plotted, for which there is good agreement between measurements and simulation, particularly for the data recorded before the installation of the DDBA cell. A small, systematic error remains for the data taken after installation, an explanation for which remains to be found. The parameters used to form the impedance model in each case are given in Table II. In order to match the bunch lengthening with current, the inductive component had to be changed from $80 \mathrm{nH}$ to $100 \mathrm{nH}$; an increase of $25 \%$.

TABLE II. Comparison of single-bunch impedance model parameters before and after the installation of the DDBA cell.

\begin{tabular}{lccc}
\hline \hline Impedance type & Parameter & Pre-DDBA & Post-DDBA \\
\hline Broadband resonator & $R_{x}$ & $50 \mathrm{k} \Omega / \mathrm{m}$ & $90 \mathrm{k} \Omega / \mathrm{m}$ \\
& $R_{y}$ & $200 \mathrm{k} \Omega / \mathrm{m}$ & $250 \mathrm{k} \Omega / \mathrm{m}$ \\
& $R_{\|}$ & $0.5 \mathrm{k} \Omega$ & $0.5 \mathrm{k} \Omega$ \\
Resistive wall & $b_{x}$ & $40.8 \mathrm{~mm}$ & $40.2 \mathrm{~mm}$ \\
Inductance & $b_{y}$ & $15.6 \mathrm{~mm}$ & $15.3 \mathrm{~mm}$ \\
\hline \hline
\end{tabular}




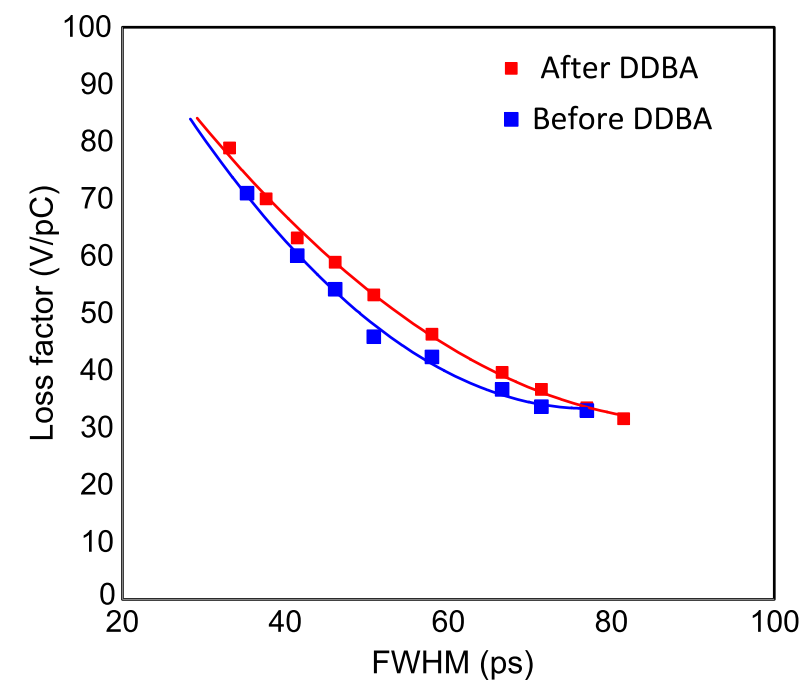

FIG. 10. Loss factor as a function of FWHM bunch length, before and after the installation of the DDBA cell.

The change in loss factor caused by the DDBA cell has been investigated by measuring the time-shift $\Delta \tau$ of the electron bunch centroid as a function of charge [30]. The loss factor $k$ can be extracted from this data using the relation

$$
k \approx 2 \pi f_{R F} \frac{e V_{R F} N_{p}}{q_{b}^{2}} \Delta \tau \cos \left(\phi_{s 0}\right)
$$

where $N_{p}$ is the number of particles per bunch, $q_{b}$ is the bunch charge and $\phi_{s 0}$ is the zero-current synchronous phase. This data is shown in Fig. 10, where the data in Fig. 9 has been used to convert $q_{b}$ into bunch length. These curves imply a rather substantial resistive component for the longitudinal impedance, above what could be anticipated from the values given in Table II. Future work aims to improve the simplified impedance model to better reflect this, in particular by replacing the purely inductive term with the full element-by-element database values.

The installation of the DDBA cell has led to an increase in the loss factor in the range 2 to $5 \mathrm{~V} / \mathrm{pC}$. This is accounted for in the model via the change in apertures used to compute the resistive wall impedance. Previous studies investigating the impact of in-vacuum IDs on single bunch instability thresholds also indicate this change is sufficient to account for differences in measured beam behavior [33].

The microwave instability threshold has been investigated by determining the bunch current at which the energy spread starts to increase, as measured using a two x-ray pinhole camera set-up [19] (see Fig. 11). These measurements were taken with 9 equally-spaced bunches in the ring in order to give sufficient intensity for the pinhole camera measurements. Despite this precaution, measurement noise clearly limits the precision with which the threshold can be

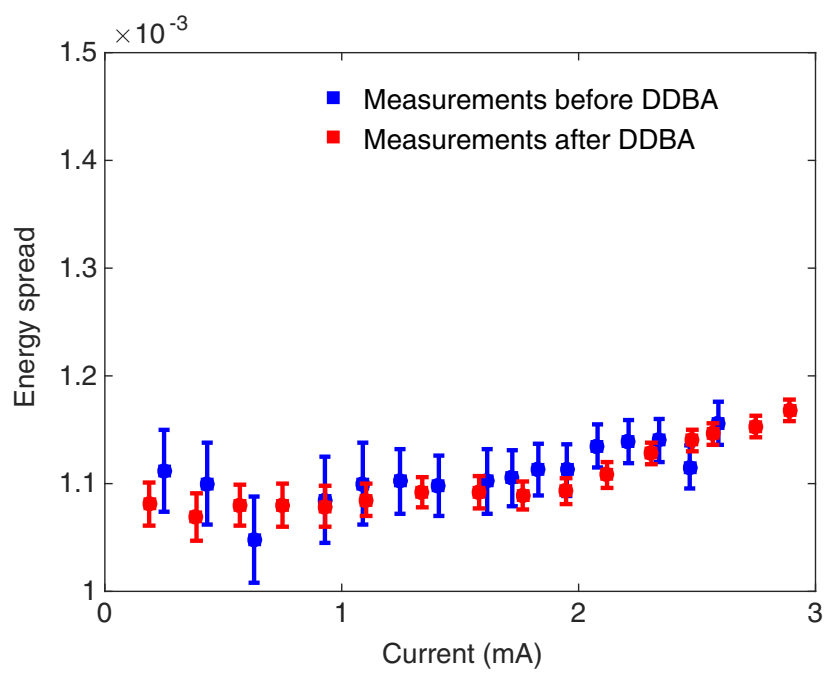

FIG. 11. Energy spread as a function of bunch current, before and after the installation of the DDBA cell.

determined. The microwave instability threshold lies in the range 1.8 to $2.0 \mathrm{~mA}$ bunch current for both data sets, with some indications that there has been a small rise in the microwave threshold after DDBA.

Single bunch instability thresholds have also been measured as a function of chromaticity in each plane. This was achieved by gradually injecting charge into a single bunch until the onset of the instability, whilst keeping the chromaticity high in the opposing plane (>3 units) [34]. A comparison of the vertical instability thresholds before and after installation is shown in Fig. 12, indicating a noticeable reduction for all chromaticity values. In order to investigated this effect further, tracking studies were carried out using SBTRACK. In this case, the only free parameter in the impedance model is the shunt

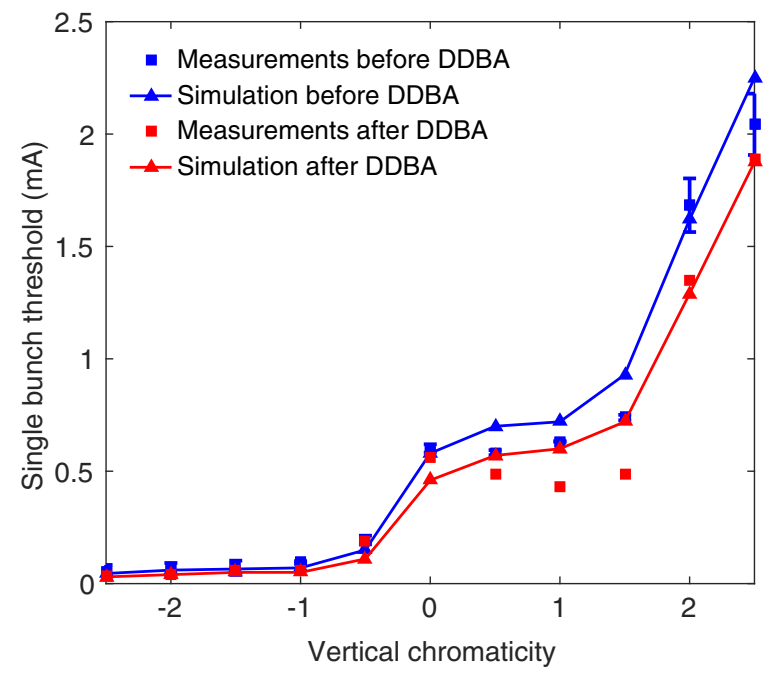

FIG. 12. Vertical instability thresholds as a function of chromaticity. 

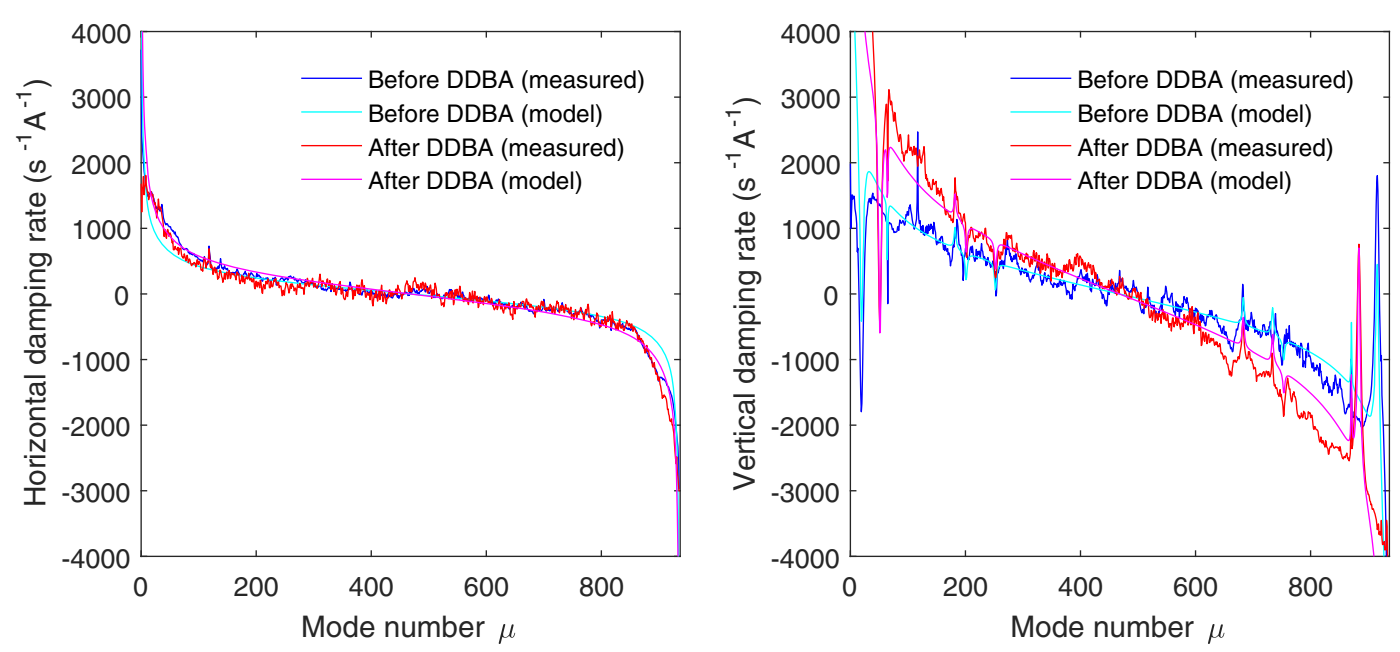

FIG. 13. Horizontal (left) and vertical (right) damping rates normalized to bunch current before and after the installation of the DDBA cell.

resistance of the broadband resonator. This was scanned, and in each case the instability thresholds were computed as a function of chromaticity, with the value that best matched the measured thresholds taken as the new reference value. As shown in Table II, the effective shunt resistance value for the ring $R_{y}$ has increased by $25 \%$ above the pre-DDBA value. Instability thresholds in the horizontal plane are generally high enough not to be of concern. However, for completeness these were also measured before and after installation and compared to simulation (data not shown). In this case, $R_{x}$ was found to have increased by almost a factor two, albeit from an initially low value.

The change in multibunch effects resulting from the DDBA cell installation has been studied by measuring the transverse damping rates as a function of mode number $\mu$ [35]. From an analytic perspective, multibunch instabilities tend to be driven by long-range wakefields arising from the RW impedance and by high-Q resonant structures formed by the vacuum chamber. Given the reduced physical apertures of the DDBA cell, the contribution from both these sources was expected to increase. Ion-related instabilities can also have an impact, but at Diamond these tend to appear only for a short period following a vacuum intervention.

In order to extract the damping rates analytically, the complex frequency shift of each mode must first be computed. Assuming a rigid Gaussian bunch structure and zero-chromaticity, the complex frequency shift is given by

$\Omega(\mu)-\omega_{\beta}=-i \frac{M N_{p} r_{e} c}{2 \gamma T_{0}^{2} \omega_{\beta}} \sum_{p=-\infty}^{\infty} Z_{x, y}\left(\omega_{\mu}\right) \exp \left(-\omega_{\mu}^{2} \sigma_{\tau}^{2}\right)$

where $\omega_{\beta}$ is the unperturbed betatron frequency, $M$ is the number of bunches, $r_{e}$ is the classical electron radius, $\gamma$ is the Lorentz factor, $T_{0}$ is the revolution period, $\sigma_{\tau}$ is the bunch length, $\omega_{\mu}$ is the oscillation frequency

$$
\omega_{\mu}=(p M+\mu) \omega_{0}+\omega_{\beta}
$$

and $\omega_{0}$ is the revolution frequency $2 \pi / T_{0}$. The damping rate for each mode is found by taking the imaginary part of Eq. (9), with the overall transverse impedance taken to be the sum of RW and narrow-band resonator impedances

$$
Z_{x, y}=Z_{x, y}^{\mathrm{RW}}+\sum Z_{x, y}^{\mathrm{res}} .
$$

Since the impedance is antisymmetric in the frequency domain, the damping rates for the multibunch oscillation modes also appears antisymmetric, with a stable mode $\mu$ also appearing as an unstable mode $h-\mu$ where $h$ is the $\mathrm{rf}$ harmonic number.

Measurements of the damping rates were carried out using the TMBF to excite each mode for 250 turns, then allowing the beam to oscillate freely for 1750 turns. This data was then used to extract the damping rate for each mode by making a linear fit to the log of the oscillation amplitude. Care was taken to ensure the bunch charge was kept uniform in all bunches (full filling pattern), and that the total beam current was low enough that the beam remained naturally stable throughout. Results for the horizontal and vertical planes before and after installation are shown in Fig. 13, in which the contribution from radiation damping has been subtracted.

For the horizontal plane, the impedance is found to be dominated by the RW component, with no significant resonant frequencies appearing. There is a good agreement with analytic calculations in each case, indicating the RW impedance has indeed been increased by the DDBA cell. For the analytic calculations, the average half-aperture was adjusted in order to give the best match to the measured 
TABLE III. Comparison of impedance model parameters before and after the installation of the DDBA cell used for multi-bunch instability studies.

\begin{tabular}{lccc}
\hline \hline Impedance type & Parameter & Pre-DDBA & Post-DDBA \\
\hline Average beta functions & $\bar{\beta}_{x}$ & $10.95 \mathrm{~m}$ & $10.24 \mathrm{~m}$ \\
& $\bar{\beta}_{y}$ & $12.25 \mathrm{~m}$ & $13.16 \mathrm{~m}$ \\
Fitted half-apertures & $b_{x}$ & $15.9 \mathrm{~mm}$ & $14.0 \mathrm{~mm}$ \\
& $b_{y}$ & $12.0 \mathrm{~mm}$ & $10.3 \mathrm{~mm}$ \\
Resonator 1 & $\left(Q_{y}, f_{y}, R_{y}\right)$ & $500,1.49 \mathrm{GHz}, 3.0 \mathrm{M} \Omega / \mathrm{m}$ & $500,1.47 \mathrm{GHz}, 3.0 \mathrm{M} \Omega / \mathrm{m}$ \\
Resonator 2 & $\left(Q_{y}, f_{y}, R_{y}\right)$ & $10000,8.96 \mathrm{GHz}, 1.4 \mathrm{M} \Omega / \mathrm{m}$ & $10000,8.96 \mathrm{GHz}, 1.4 \mathrm{M} \Omega / \mathrm{m}$ \\
Resonator 3 & $\left(Q_{y}, f_{y}, R_{y}\right)$ & $500,1.10 \mathrm{GHz}, 0.3 \mathrm{M} \Omega / \mathrm{m}$ & $500,1.10 \mathrm{GHz}, 0.3 \mathrm{M} \Omega / \mathrm{m}$ \\
Resonator 4 & $\left(Q_{y}, f_{y}, R_{y}\right)$ & $3000,6.86 \mathrm{GHz}, 0.5 \mathrm{M} \Omega / \mathrm{m}$ & $3000,6.86 \mathrm{GHz}, 0.5 \mathrm{M} \Omega / \mathrm{m}$ \\
Resonator 5 & $\left(Q_{y}, f_{y}, R_{y}\right)$ & $3000,6.89 \mathrm{GHz}, 0.5 \mathrm{M} \Omega / \mathrm{m}$ & $3000,6.89 \mathrm{GHz}, 0.5 \mathrm{M} \Omega / \mathrm{m}$ \\
\hline \hline
\end{tabular}

data, the results of which are given in Table III. The fitted values for the half-apertures are significantly smaller than those calculated from the engineering drawings and given in Table II, an explanation for which remains to be found.

In the vertical plane several peaks appear in the data in addition to the RW contribution. The dominant peaks at modes 19 (before DDBA) and 51 (after DDBA) have been identified as being driven by the vertical collimator, with the change in mode number due to a realignment of the collimator blades between the measurements. The remaining peaks have remained fixed in frequency and amplitude, implying no new significant narrowband sources have been introduced.

\section{F. Impact on orbit stability}

The dominant cause of electron beam motion is vibrations of the quadrupole magnets. The impact that an individual magnet has on the beam position depends upon the locations of the magnet and observation points $(s)$, the integrated strength $\left(K_{q} L_{q}\right)$ and the size of the displacement $(\Delta x)$, and is given by

$$
\begin{aligned}
z(s)= & \left(\frac{\sqrt{\beta(s) \beta_{q}}}{2 \sin \left(\pi Q_{z}\right)} \cos \left(\left|\phi(s)-\phi_{q}\right|-\pi Q_{z}\right)\right. \\
& \left.+\frac{\eta_{z}(s) \eta_{z, q}}{\alpha_{c} L_{q}}\right) K_{q} L_{q} \Delta x
\end{aligned}
$$

where $z$ refers to either the horizontal or vertical plane, $\phi$ and $\beta$ are the betatron phase and amplitude, $Q_{z}$ is the betatron tune, $\eta_{z}$ is the dispersion and $\alpha_{c}$ is the momentum compaction factor. Typically the first term on the right hand side of Eq. (12) dominates, and the presence of the $1 / \sin (\pi Q)$ term indicates that a $20 \%$ increase in beam motion can be expected for the new lattice in the vertical plane simply due to the change in tune point. Since the gradient of the new quadrupoles in the DDBA cell is much higher than in the standard DBA magnets it could be anticipated that they will have a larger impact on orbit stability. However, this increase in strength is offset by the lower beta-functions at their location.

The original goal for Diamond was that the electron beam position should be stable to $10 \%$ of the beam size at the ID source points in the $1-100 \mathrm{~Hz}$ band width. As part of the long-term strategy, this goal has now been extended to provide a stability of $3 \%$ of beam size in the range $1-1000 \mathrm{~Hz}$ in recognition of the improvement in beam-line detector resolution and acquisition rate. Since Diamond operates with $8 \mathrm{pm}$ rad vertical emittance, this new target translates to an improvement in absolute stability from $0.35 \mu \mathrm{m}$ to $0.1 \mu \mathrm{m}$ in the centre of a standard ID straight in the vertical plane.

The orbit stability at the ID source points is shown in Fig. 14. The beam motion is found to have increased cell by $40 \%$ in the $1-100 \mathrm{~Hz}$ bandwidth and $50 \%$ in the $1-1000 \mathrm{~Hz}$ range, on average. However, the exact size of the increase is found to depend upon the location. This indicates that the

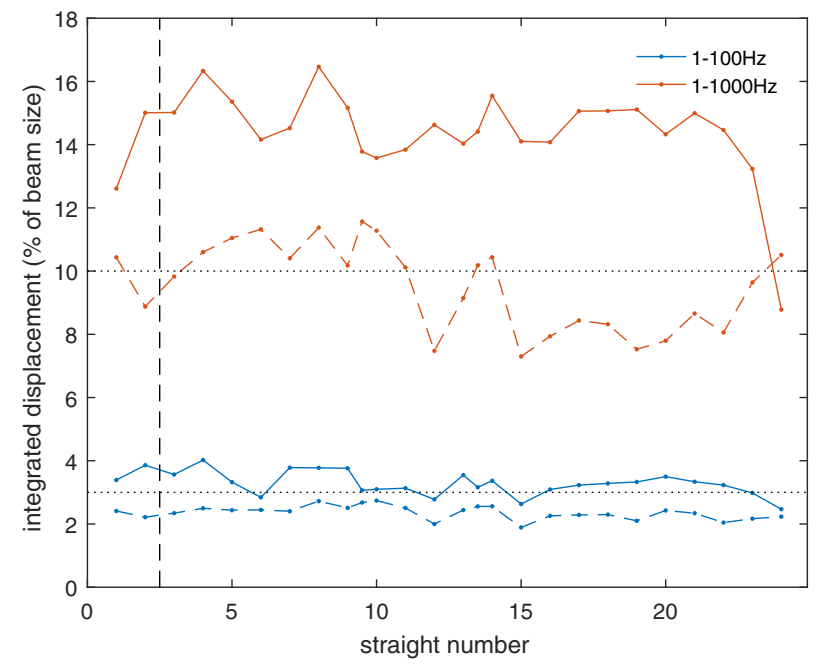

FIG. 14. Vertical orbit motion in the straight sections as a percentage of beam size. Data recorded before (dashed colored lines) and after (solid colored lines) the installation of the DDBA cell (vertical dashed black line) are shown. Fast orbit feedback was running during the measurements. 


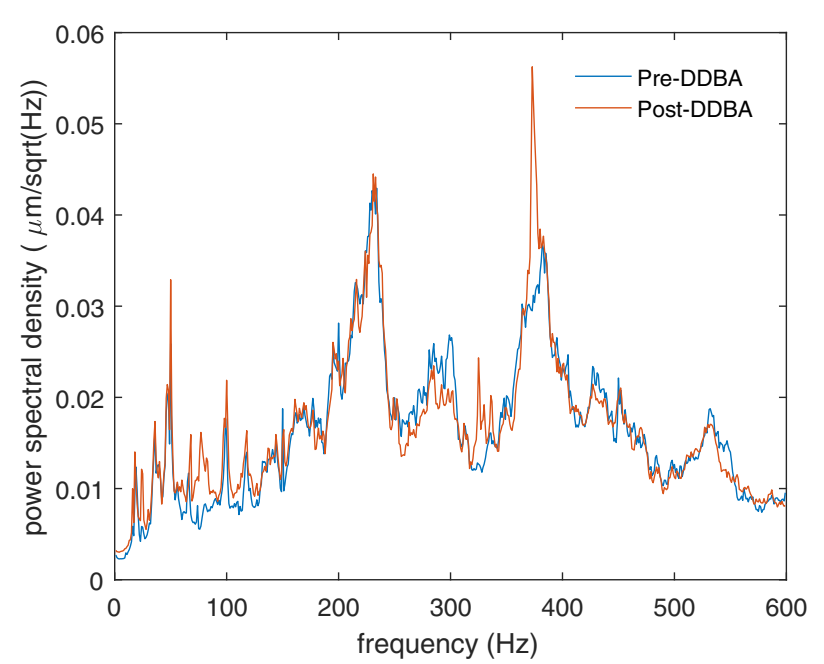

FIG. 15. Vertical power spectral density normalized to $\beta_{y}=1.5 \mathrm{~m}$. The spectrum remains largely unchanged in shape, with new features appearing around $75 \mathrm{~Hz}, 325 \mathrm{~Hz}$ and $375 \mathrm{~Hz}$.

vibration of the magnets is not uniform everywhere, and that the phase advance from source to observation point plays a role.

A common way of expressing the beam motion is by using the power spectral density (PSD), which for discrete time steps of $\Delta t$ and a total acquisition time of $T=N \Delta t$ is defined as

$$
S(\omega)=\frac{(\Delta t)^{2}}{T}\left|\sum_{n=1}^{N} z_{n} e^{-i \omega n}\right|^{2}
$$

where $\omega$ is the angular frequency and $z_{n}$ is the beam position at time $t=n \Delta t$.

In order to compare the beam spectrum before and after the installation, PSDs have been calculated by normalizing the data from all BPMs to $\beta_{y}=1.5 \mathrm{~m}$ and averaging around the ring to produce a single spectrum. This is shown in Fig. 15. Overall, very few new features have appeared in the spectrum. The amplitude is generally raised at all frequencies, with a line at $50 \mathrm{~Hz}$ particularly prominent. Additionally, new peaks are observed around $75 \mathrm{~Hz}, 325 \mathrm{~Hz}$ and $375 \mathrm{~Hz}$.

In order to identify the source of the new features, a quadrupole displacement to beam position response matrix was constructed using Eq. (12). This was inverted using singular value decomposition and then multiplied by the individual BPM spectra, analogous to the calculation performed by the regular orbit feedback applications. The results are shown in Fig. 16, in which the DDBA cell is highlighted with white dashed lines.

There are three main frequency bands which are responsible for generating the majority of orbit motion. The first of these is below $50 \mathrm{~Hz}$, within which there are many discrete frequencies appearing. These have been previously identified as being driven by numerous items such as

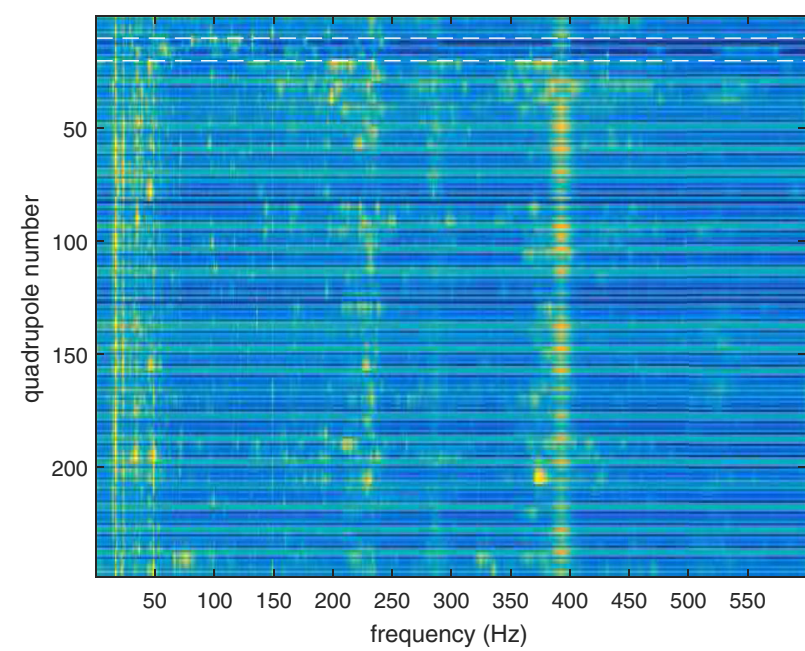

FIG. 16. Projected quadrupole motion as a function of frequency and position in the ring. The data has been calculated using the raw BPM data and assumed to be caused purely by quadrupole displacement. The location of the DDBA cell has been highlighted using white dashed lines, and the color scaling shows the change in amplitude in arbitrary units.

cooling water pumps and air handling units. The other two bands occur at around $230 \mathrm{~Hz}$ and $380 \mathrm{~Hz}$. These are known to be caused by turbulent flow in the magnet cooling water which coincide with mechanical resonances of the system. Work is underway to reduce the rate of cooling water flow and remove these vibrations at source [36]. The contribution from the DDBA cell appears to be small, with the amplitude of magnet vibrations visibly lower than elsewhere in the ring, with the exception of a few lines around $60-120 \mathrm{~Hz}$ (particularly for girder 1). The new features identified in Fig. 15 at $75 \mathrm{~Hz}$ and $325 \mathrm{~Hz}$ appear to originate in cell 24 (quadrupoles 239 to 243), and the line at $375 \mathrm{~Hz}$ looks to be driven by magnets in cell 20 (quadrupoles 203 to 208) and are unrelated to the installation of the DDBA cell.

\section{CONCLUSIONS}

The replacement of a single DBA cell with a DDBA structure was successfully completed in November 2016, thereby creating space for the installation and operation of a new $2 \mathrm{~m}$ long in-vacuum insertion device. The new cell has been designed with many features in common with modern multi-bend achromat lattices, including strong focussing magnets, gradient bends and small vacuum chamber apertures. The installation has led to a strong break in the machine symmetry, requiring a careful reoptimization of both the linear and nonlinear storage ring optics. The experience gained from the DDBA upgrade at Diamond demonstrates that ambitious lattice modifications can be achieved, and that good lifetime and injection efficiency can be maintained when the periodicity is reduced to one. 
The results contained in this article demonstrate the strengths and limitations of using standard modelling codes to predict the performance of such a substantial lattice modification. As can be expected, excellent control of the linear optics has been maintained, with the match to the machine model at the $1 \%$ level and correction to below $2 \mathrm{pm}$ rad vertical emittance straightforward. Dynamic aperture, frequency map, and nonlinear chromaticity measurements show good agreement with model predictions, however, a stronger than expected resonance is clearly limiting the horizontal dynamic aperture and hence the injection efficiency. Small discrepancies in the vertical detuning with energy and amplitude also remain to be understood. There is excellent agreement for the injection efficiency tune maps and lifetime as a function of rf voltage curves, requiring only relatively minor scaling to bring the model in line with measurements.

Studies of collective effects such as bunch lengthening with current, single bunch instability thresholds and multi-bunch damping rates have highlighted the impact that the new vacuum chamber in the DDBA cell has had on the machine impedance. This section of the ring is of a relatively complex structure, consisting of a mix of copper and stainless steel narrow-gap sections (13.5 $\mathrm{mm} \times 9 \mathrm{~mm}$ internal half-aperture), with a number of discrete inner wedges that are used to absorb synchrotron radiation at high loss points [11]. By using a simplified impedance model to reproduce the measurements, it has been possible to quantify the increase in inductance, resistive wall impedance, and effective BBR shunt impedance that this has had. Whilst there has not been a significant impact in the standard operation of the existing storage ring, the implications for a full-ring upgrade are clear. The design of a low-impedance vacuum chamber will be one of the most significant challenges for new rings, for example through the use of low resistivity materials such as copper, ante-chambers for better heat load management, shallow tapered sections and the inclusion of a multibunch feedback for damping the resulting instabilities.

\section{ACKNOWLEDGMENTS}

The authors would like to thank T. Pulampong and A. Shahveh for input and discussions on the lattice and magnet design work, G. Rehm, A. Morgan and L. Bobb for expert support with the diagnostic equipment, and members of the Operations group for assistance during data acquisition. J. Bengtsson is also thanked for many stimulating discussions on modelling techniques. One of the authors (RB) has received funding from the European Unions Horizon 2020 Research and Innovation programme under Grant Agreement No. 730871.
[1] D. Robin, J. Safranek, and W. Decking, Realizing the benefits of restored periodicity in the advanced light source, Phys. Rev. ST Accel. Beams 2, 044001 (1999).

[2] D. Robin et al., in Proceedings of the 8th European Particle Accelerator Conference, Paris, 2002 (EPS-IGA and CERN, Geneva, 2002), p. 215.

[3] H. Nishimura and D. Robin, in Proceedings of the 18th Particle Accelerator Conference, New York, 1999 (IEEE, New York, 1999), p. 203.

[4] A. Gabard, D. George, M. Negrazus, L. Rivkin, V. Vrankovic, Y. Kolokolnikov, and P. Vobly, in Proceedings of the 2nd International Particle Accelerator Conference, San Sebastián, Spain (EPS-AG, Spain, 2011), p. 3038.

[5] G. Ingold, A. Streun, B. Singh, R. Abela, P. Beaud, G. Knopp, L. Rivkin, V. Schlott, T. Schmidt, H. Sigg, J. van der Veen, A. Wrulich, and S. Khan, in Proceedings of the 19th Particle Accelerator Conference, Chicago, IL, 2001 (IEEE, Piscataway, NJ, 2001), p. 2656.

[6] Diamond Synchrotron Light Source: Report of the Design Specification, CCLRC Report, 2002.

[7] R. Walker, in Proceedings of Asian Particle Accelerator Conference (ACFA, Indore, India, 2007), p. 66.

[8] Z. Patel, E. Rial, A. George, S. Milward, A. Rose, R. P. Walker, and J. Williams, in Proceedings of International Particle Accelerator Conference (EPS-AG, Copenhagen, Denmark, 2017), p. 1592.

[9] B. Singh, R. Fielder, I. Martin, G. Rehm, and R. Bartolini, in Proceedings of the 2nd International Particle Accelerator Conference, San Sebastián, Spain (EPS-AG, Spain, 2011), p. 2103.

[10] R. Walker, in Proceedings of the 5th International Particle Accelerator Conference (EPS-AG, Dresden, Germany, 2014), p. 331.

[11] R. Bartolini et al., Double-double bend achromat cell upgrade at the Diamond Light Source: From design to commissioning, Phys. Rev. Accel. Beams 21, 050701 (2018).

[12] R. Bartolini and T. Pulampong, in Proceedings of the 4th International Particle Accelerator Conference, IPAC2013, Shanghai, China, 2013 (ACFA/EPS-AG/APSDPB, Shanghai, China, 2013), p. 237.

[13] P. Tavares, S. Leemann, M. Sjöström, and Å. Andersson, The MAX IV storage ring project, J. Synchrotron Radiat. 21, 862 (2014).

[14] ESRF Upgrade Programme Phase II (Orange Book), ESRF Technical Design Report, 2014.

[15] L. Liu, N. Milas, A. Mukai, X. Resende, and F. de Sá, The Sirius project, J. Synchrotron Radiat. 21, 904 (2014).

[16] R. Tomás, M. Aiba, A. Franchi, and U. Iriso, Review of linear optics measurement and correction for charged particle accelerators, Phys. Rev. Accel. Beams 20, 054801 (2017).

[17] J. Safranek, Experimental determination of storage ring optics using orbit response measurements, Nucl. Instrum. Methods Phys. Res., Sect. A 388, 27 (1997).

[18] I. Martin, M. Abbott, M. Furseman, G. Rehm, and R. Bartolini, in Proceedings of the 5th International Particle Accelerator Conference, Dresden, Germany, 2014 (EPSAG, Dresden, Germany, 2014), p. 1763. 
[19] C. Thomas, G. Rehm, I. Martin, and R. Bartolini, X-ray pinhole camera resolution and emittance measurement, Phys. Rev. ST Accel. Beams 13, 022805 (2010).

[20] R. Bartolini, I. Martin, G. Rehm, and F. Schmidt, Calibration of the nonlinear ring model at the Diamond Light Source, Phys. Rev. ST Accel. Beams 14, 054003 (2011).

[21] M. Borland, elegant: A flexible SDDS-compliant code for accelerator simulation, Report No. LS-287, 2000.

[22] I. Martin, M. Apollonio, R. Fielder, G. Rehm, and R. Bartolini, in Proceedings of the 2nd International Particle Accelerator Conference, San Sebastin, Spain (EPS-AG, Spain, 2011), p. 1404.

[23] J. Bengtsson, I. Martin, J. Rowland, and R. Bartolini, Online control of the nonlinear dynamics for synchrotrons, Phys. Rev. ST Accel. Beams 18, 074002 (2015).

[24] A. Piwinski, The Touscheck effect in strong focussing storage rings, DESY Report No. DESY 98-179, 1998.

[25] C. Steier, D. Robin, L. Nadolski, W. Decking, Y. Wu, and J. Laskar, Measuring and optimizing the momentum aperture in a particle accelerator, Phys. Rev. E 65, 056506 (2002).

[26] S. Leemann, Interplay of Touschek scattering, intrabeam scattering, and $\mathrm{rf}$ cavities in ultralow-emittance storage rings, Phys. Rev. ST Accel. Beams 17, 050705 (2014).

[27] A. Andersson and A. Streun, in Proceedings of the 10th European Particle Accelerator Conference, Edinburgh, Scotland, 2006 (EPS-AG, Edinburgh, Scotland, 2006), p. 3421.

[28] V. Smaluk, I. Martin, R. Fielder, and R. Bartolini, Beambased model of broad-band impedance of the Diamond
Light Source, Phys. Rev. ST Accel. Beams 18, 064401 (2015).

[29] R. Nagaoka, J. Rowland, and R. Bartolini, in Proceedings of the 23rd Particle Accelerator Conference, Vancouver, Canada, 2009 (IEEE, Piscataway, NJ, 2009), p. 4637.

[30] E. Koukovini-Platia, L. Bobb, R. Fielder, and R. Bartolini, in Proceedings of the 8th International Particle Accelerator Conference, Copenhagen, Denmark, 2017 (EPS-AG, Copenhagen, Denmark, 2017), p. 4493.

[31] R. L. Gluckstern, J. van Zeijts, and B. Zotter, Coupling impedance of beam pipes of general cross section, Phys. Rev. E 47, 656 (1993).

[32] L. Bobb, A. Morgan, and G. Rehm, in Proceedings of the 4th International Beam Instrumentation Conference (JACoW, Melbourne, Australia, 2015), p. 206.

[33] E. Koukovini-Platia, M. Apollonio, R. Bartolini, R. Fielder, and I. Martin, in Proceedings of the 7th International Particle Accelerator Conference, Busan, Korea, 2016 (ACFA/EPS-AG/APS-DPB, Busan, Korea, 2016), p. 637.

[34] E. Koukovini-Platia, A. Morgan, G. Rehm, and R. Bartolini, in Proceedings of the 8th International Particle Accelerator Conference, Copenhagen, Denmark, 2017 (EPS-AG, Copenhagen, Denmark, 2017), p. 4489.

[35] R. Bartolini, R. Fielder, E. Koukovini-Platia, and G. Rehm, in Proceedings of the 8th International Particle Accelerator Conference, Copenhagen, Denmark, 2017 (EPS-AG, Copenhagen, Denmark, 2017), p. 4485.

[36] G. Rehm and N. Hammond (private communication). 